\title{
TTR
}

Traduction, terminologie, re?daction

\section{Écriture de soi et traduction dans les oeuvres " jumelles " de Samuel Beckett et Wilfred R. Bion}

\section{Simon Harel}

Volume 11, numéro 2, 2e semestre 1998

Psychanalyse et traduction : voies de traverse

Psychoanalysis and Translation: Passages Between and Beyond

URI : https://id.erudit.org/iderudit/037339ar

DOI : https://doi.org/10.7202/037339ar

Aller au sommaire du numéro

Éditeur(s)

Association canadienne de traductologie

ISSN

0835-8443 (imprimé)

1708-2188 (numérique)

Découvrir la revue

Citer cet article

Harel, S. (1998). Écriture de soi et traduction dans les oeuvres « jumelles » de Samuel Beckett et Wilfred R. Bion. TTR, 11(2), 153-184.

https://doi.org/10.7202/037339ar
Résumé de l'article

Écriture de soi et traduction dans les oeuvres « jumelles » de Samuel Beckett et Wilfred R. Bion - Cet article envisage la problématique de la traduction à partir du champ psychanalytique. Wilfred R. Bion fut pour une brève période l'analyste de Samuel Beckett. L'auteur souhaite démontrer que les écrits de Samuel Beckett et Wilfred R. Bion peuvent être lus comme des oeuvres jumelles qui tentent à leur manière de traduire l'infigurable source du " secret » de la cure. L'auteur aborde l'étude du concept d'après coup en psychanalyse, la mise enjeu du mythe de Babel dans l'oeuvre théorique de Bion, la traduction de la langue-mère dans l'oeuvre de Beckett. Les oeuvres de Bion et Beckett tenteraient ainsi l'impossible traduction du trauma dans la langue maternelle.
Tous droits réservés ( $\mathrm{TTR}$ : traduction, terminologie, rédaction - Les auteurs, 1998 cest protégé par la loi sur le droit d’auteur. L'utilisation des services d'Érudit (y compris la reproduction) est assujettie à sa politique d'utilisation que vous pouvez consulter en ligne.

https://apropos.erudit.org/fr/usagers/politique-dutilisation/ 


\section{Écriture de soi et traduction dans les œuvres " jumelles" de Samuel Beckett et Wilfred R. Bion}

\section{Simon Harel}

\section{L'écriture du trauma}

Les écritures croisées de Samuel Beckett et de Wilfred Ruprecht Bion témoignent à certains égards de l'inscription de la matérialité du processus analytique. C'est le propos que je voudrais développer, faisant valoir que la coénonciation apparaît dans ces cuvres comme la mise au jour d'un jumeau imaginaire qui incame les apories de la traduction du monde inconscient. Je mettrai l'accent sur la constitution de l'écriture autofictionnelle de Wilfred $R$. Bion afin d'indiquer ce qu'elle doit à l'analysant que fut pour un temps Samuel Beckett. Pour l'analyste comme pour l'écrivain, la posture adoptée face au désir de savoir est complexe. L'écrivain peut rêver de détenir le secret de son cuvre. Ce faisant, il adopte une attitude malaisée qui le situe tout à la fois en amont et en aval de l'œuvre créée. Comment l'écrivain pourrait-il posséder ce secret, le faire sien, si ce n'est au prix d'une impossible traduction qui le déclarerait récipiendaire de son inconscient? La littérature est précisément cet objet dénaturé, ouvert à la démesure des codes qui formulent les conditions du passage à la fiction. Il est impossible pour l'écrivain d'anticiper le moment d'émergence de sa passion pour la chose littéraire. Pour de singulières raisons, la psychanalyse rencontre la littérature sur ce terrain. Celle-ci admet que le discours est sans cesse heurté, brisé, biffé, en somme que l'énonciation littéraire révèle l'emprise négative d'une 
passion qui bute sur le presque-rien. Celle-là admet que le sujet se dit dans la violence quotidienne d'un silence d'où sourd une parole soigneusement malmenée par la résistance. Dans les deux cas, le sujet est hanté par la figure d'une traduction pleine et entière de soi.

Voilà pourquoi la traduction, dès qu'on l'aborde de l'intérieur du champ psychanalytique, est réfractaire à toute forme de contrôle de la discursivité. L'acte de traduire ne signifie pas l'affirmation d'une vision unificatrice qui octroierait au savoir harmonie et cohérence. L'acte de traduire impose plutôt la rencontre du trauma qui est la déformation violente d'un savoir constitué.

Les définitions du trauma sont nombreuses et variées. Nous retiendrons, pour les fins de cet article, l'idée d'une charge pulsionnelle dont la source endopsychique est si violente qu'elle impose au sujet d'avoir oublié ce qui a fait l'objet du refoulement. Cette définition est commode et sans doute trop générale. Elle possède cependant l'immense avantage d'indiquer la part d'infigurable qui nourrit le trauma. C'est en effet une défaillance de l'acte de traduction qui interdit la mise au jour préconsciente et consciente du trauma. On pourrait avancer à cet égard que le trauma se constitue à la suite de l'autodestruction des paramètres sémiotiques de l'appareil psychique. Incapable de traduire la poussée de l'énergie pulsionnelle, le sujet est placé devant l'obligation de recevoir ce déferlement massif que Bion avait nommé avec beaucoup de justesse la " turbulence émotionnelle ${ }^{l}$ ". Nous retiendrons donc de cette définition du trauma la mise au jour d'une tache aveugle qui résiste obstinément à la figuration consciente. La psychanalyse, lorsqu'elle rencontre la dure logique du trauma, ne peut elle-même que demeurer silencieuse et discrète. Toute tentative, aussi opportune et délicate qu'elle puisse paraître, de favoriser l'émergence d'une pensée pouvant penser le trauma, est un labeur difficile. Sans doute s'agit-il dans le meilleur des cas de restituer au sujet une capacité sémiotique de traduction de son for intérieur. L'enjeu est complexe puisque cet acte de traduction se situe au carrefour de deux mondes : la temporalité préconsciente/consciente obéit

\footnotetext{
' Wilfred R. Bion, * Emotional Turbulence *, conférence lue dans le cadre de "The International Conference on Borderline Disorders ", Topeka, Kansas (March 1976), publié dans Clinical Seminars and Four Papers, Abingdon, Fleetwood Press, 1987.
} 
à des paramètres d'investigation qui appartiennent au monde linéaire de la narrativité. L'acte de traduction suppose que le sujet est en mesure de maintenir une tension discursive entre la linéarité du fait narratif et la dimension proprement sémiotique et tabulaire de l'inconscient.

Cette capacité sémiotique de traduction que $\mathrm{j}$ 'évoque rappelle l'importance de la temporalité préconsciente/consciente. Cette dernière, faut-il le préciser, ne correspond pas à une vision du monde. L'association du langage au monde préconscient/conscient ne signifie pas que le sujet circule dans un univers dont la complétude est la caractéristique première. Ce point de vue, qui souscrit à une compréhension euphorique et unitaire du monde langagier, pose problème pour plusieurs raisons. Le monde du langage n'est pas cette heureuse demeure qui situe le sujet à l'écart de l'histoire, de ses turbulences individuelles et de ses défaillances collectives. Voilà pourquoi la psychanalyse ne saurait souscrire, s'agissant du travail de la traduction, à cet idéal du retour au monde natal de la complétude maternelle. Cet idéal peut animer l'activité langagière. Il faut alors noter qu'il s'agit d'un projet dont la démesure forge l'emprise du Surmoi. On ne saurait oublier de quelle façon la culpabilité et la honte, toutes deux liées à l'instauration précoce du Surmoi, contribuent à l'édification contraignante d'un langage condamné à quitter l'heureuse demeure du monde maternel.

L'antinomie que je dessine à grands traits a pour but de faire valoir l'indispensable complémentarité de la fusion et de la destruction au cœur du travail de la traduction. Le sujet qui habite le monde préconscient/conscient du langage croit loger dans une paisible demeure. Il garde à l'esprit cette conviction intime qui lui permet de nouer une relation de proximité avec l'énoncé dont il déclare être le propriétaire. Sans doute est-il juste d'entrevoir dans cette allusion imaginaire la certitude d'un sentiment d'identité qui nourrit l'énonciation. Il n'en reste pas moins que ce sentiment de proximité est aussi une illusion dont le Surmoi se nourrit afin de mieux faire valoir la démesure de l'Idéal. Traduire, on l'aura compris, est une tâche impossible. La psychanalyse s'y reconnaît puisque l'écoute qu'elle promeut appartient à ce registre tabulaire proche de la diction du rêve, forme rigoureuse de l'inconscient.

Revenons à cette interrogation qui aborde la diction du trauma. Ce demier ne trouve matière à énonciation que par l'infigurable qui est sa 
raison d'être. Façon de dire que le trauma ne peut être l'objet d'une énonciation lénifiante. Le trauma n'est pas seulement ce qui résiste inlassablement à la mise en discours. Il est plus fondamentalement au cœur de la discursivité, ce silence précieusement entretenu sur l'origine du langage. Voilà pourquoi le trauma ne saurait se payer de mots... Par le silence démesuré qu'il contribue à laisser entendre, le trauma interroge la source de l'infigurable.

L'œuvre de Beckett peut représenter sous cet angle une diction du trauma qu'il convient d'examiner. Le trauma est cet arrachement mis en jeu par les mots; c'est du moins une définition que requiert la littérature lorsqu'elle prétend nommer la source du trauma. Beckett aura tenté sans relâche de nommer cette brutale contestation du langage. C'est d'ailleurs parce que les mots ne suffisent plus que la littérature est vitale. Parce qu'il est espéré que le trauma soit nommé, la littérature doit faire appel aux mots du désordre afin de dire cette cassure de la mémoire qui appartient à un monde inconscient fermé sur lui-même. Beckett aura été à cet égard un écrivain fascinant. $\grave{A}$ la fois silencieux et prodigieusement bavard, il aura révélé par une écriture rebelle et désordonnée l'envers du trauma. Par une violente torsion dont il est difficile de comprendre l'action, Beckett aura écrit avec les maux de la solitude et de la mort, cet infigurable dont le trauma est la forme avérée. Le psychanalyste Wilfred R. Bion aura été fasciné par cet hormme-là puisque Beckett témoignait de façon spectaculaire d'un changement radical de vertex. Circulant librement du monde inconscient au détroit de la parole que dicte la création littéraire, Beckett aura offert l'image sans doute paradoxale d'une écriture à la fois muette et sûre d'elle-même.

Ce changement de vertex, qui témoigne de cette alternance du silence et de l'élocution littéraire, Bion, de son côté, en cherchait activement la source dans la déliaison de la pensée psychotique. C'est encore une fois de turbulence émotionnelle - pour reprendre l'expression de Bion - qu'il s'agit ici. Comment arriver à dire, à moins qu'il s'agisse de laisser se dire le trauma?

Dans ce domaine, toute expression volontaire, forcée, ne peut mener qu'à l'échec puisque les mots poursuivent leur ronde solitaire, peu soucieux d'être transmis à autrui. Le psychanalyste connaît ce parcours puisque l'une de ses tâches est d'épuiser par un silence respectueux une 
parole qui accueille l'épreuve négative du trauma. Quant à la littérature, elle n'ignore pas ce parcours qui transforme l'infigurable en registre d'expression. Elle ne saurait surtout ignorer que l'on n'arrive pas à surmonter le trauma. La diction de l'infigurable n'est pas cette quête victorieuse qui permet de contrer l'épreuve négative du trauma. Il faudrait plutôt imaginer une façon * autre "d'écrire le trauma : un acte naratif échappant à l'anamnèse, peu soucieux de la chronologie des faits.

Bion formulera de nombreuses années après la terminaison de l'analyse avec Beckett cette remarque lumineuse : l'analyse n'est ni mémoire ni désir'. Sous l'apparente ascèse de la formulation, que faut-il entendre au plus près? J'ai le sentiment que Bion cherchait à faire surgir cette résonance du silence - source du trauma - au cour de la parole. La coïncidence est troublante. Beckett $s$ 'acharne a trouver les mots qui dans une langue étrangère creusent cette fosse de solitude et de désarroi qu'il est possible d'associer au trauma. Bion, de son côté, renouvelle les paramètres de l'écoute analytique en proposant un langage qui ignore le signe secondaire de la narration, soucieux de laisser se dessiner la forme brouillée de cet infigurable.

Beckett représenta peut-être pour Bion cette emprise du trauma qu'il était loisible de * situer " afin qu'un sujet-psychanalyste s'en fasse l'interprétant. Encore qu'il soit nécessaire de nuancer ce propos. Ce n'est pas Bion qui tente de forcer la singularité d'une emprise transférentielle dont la source traumatique aurait été patente. Pas plus que Beckett, à la faveur de l'analyse avec Bion, ne tente de savoir comment son ouvre aurait pu loger à rebours une théorisation dont il serait demeuré l'agent involontaire. Je n'entends pas ici décréter que le déroulement de la cure avec Bion aurait permis le déploiement d'une pensée créative chez Beckett. Pas plus que je n'affirme que l'œuvre à venir de Beckett aurait contenu pour Bion la source pulsionnelle d'un savoir théorique qui se serait matérialisé des années plus tard.

Beckett et Bion ont été fidèles à cette règle nécessaire de la confidentialité. On sait que la confidentialité témoigne de la valeur

${ }^{2}$ Wilfred R. Bion, * Notes on Memory and Desire ", The Psychoanalytic Forum (Los Angeles, California), 2 (3). 
attribuée au secret. Pour nous, lecteurs de Beckett et de Bion, cette confidentialité a un sens. Elle contribue à mettre en perspective cet infigurable auquel je faisais référence un peu plus tôt. Beckett et Bion auront été silencieux sur l'épisode de la cure. Ils auront refusé de céder la parole à l'illusion autobiographique ou à la déclamation fictionnelle de l'expérience analytique. C'est le maintien de ce secret qui permet l'émergence de notre pensée. Il s'agit bien ici, encore une fois, d'un infigurable, mais d'un infigurable heureux. La confidentialité nous permet d'aménager un espace de pensée propre. Plus encore, cette confidentialité nous permet de proposer l'invention de la relation Bion/Beckett, sans crainte de favoriser une inutile justification psychobiographique.

Je n'entends pas valoriser par cette expression une vertueuse nécessité qui condamne au silence parce qu'il faut bien respecter la parole du patient. Il est bien sûr impératif que l'analyste sache se taire... Ce qui ne signifie pas par ailleurs que la psychanalyse soit condamnée au silence et à la culpabilité surmolque. L'analyse n'est pas, dans la perspective que j'explore ici, ce cloisonnement étanche entre le secret de la cure et une parole publique désordonnée, habitée par le spectre de la dénonciation, nourtie par l'oralité de la rumeur anonyme. L'espace analytique, tel que je le mets en scène à la faveur de cet article, échappe à ce cloisonnement par la mise en jeu du travail de la fiction. N'oublions pas que Murphy, premier véritable roman de Beckett, est contemporain de l'analyse avec Bion. N'oublions pas que Bion fera appel à la fiction afin de traduire la singularité de l'expérience analytique. Chez Bion, le projet autofictionnel qu'est $A$ Memoir of the Future consiste à briser l'unité de la voix narrative. À l'occasion de cette trilogie, l'auteur Bion devient personnage, narrateur, incarnation ironique d'un protagoniste nommé P.A., ou encore témoin survivant de la bataille d'Yprès au cours de la guerre de 19141918. Le passage à la fiction est ici majeur puisque Bion tente de donner corps à cet infigurable de l'expérience analytique dont il soutient à la même époque qu'elle doit être sans mémoire ni désir. Bion et Beckett ont

${ }^{3}$ Wilfred R. Bion, A Memoir of the Future. Book One : The Dream (1975). Book Two : The Past Presented (1977), Brazil, Imago Editoria. Book Three : The Dawn of Oblivion (1979), Pertshire, Clunie Press. 
été saisis à divers degrés par cette emprise de la fiction qui leur imposait de s'écrire pour mieux devenir les protagonistes multiples de leurs existences.

Comment expliquer alors que le motif de la traduction impose chez Bion et Beckett une pensée de l'infigurable? Beckett écrira en français une cuvre dont le déferlement créateur fait appel à une langue étrangère. L'exigence de traduction est ici immédiate puisqu'elle semble permettre l'accueil de représentations de choses dont la symbolisation apparaissait particulièrement ardue dans la langue maternelle. Bion de son côté inscrira la traduction comme motif organisateur de l'appareil psychique bien qu'il emploie rarement cette expression de manière manifeste. Il n'en demeure pas moins que l'exigence de traduction est pensée chez Bion comme la forme actuelle du travail psychique. L'acte de trouver un contenant pour des pensées non symbolisées appartient à ce registre de traduction où les représentations de choses doivent être accueillies dans un corps-langage. L'opération ne peut être ici cathartique et purgative. Le corps est perçu comme la forme sémiotique d'un contenant psychique qui accueille les représentations de choses afin de les transformer en contenus émotionnels qui peuvent à leur tour être pensés. Pour Bion, l'exigence de traduction recourt à des paramètres sémiotiques puisqu'il s'agit de penser la transformation de la source pulsionnelle selon qu'elle prenne la forme d'un élément-alpha, d'un élément-bêta, de pensées du rêve, d'une préconception, ou encore d'une conception.

Une pensée de la rupture accompagne l'œuvre de Bion. Cette rupture prend forme grâce à l'intervention de l'identification projective : processus primaire qui structure les premières rencontres du monde psychique avec l'altérité externe. Cette pensée de la rupture chez Bion correspond à la tentative d'aménager un contenant psychique - une source de contenance - permettant de loger une turbulence émotionnelle qui est à la source de l'acte de penser. Quant à Beckett, cette mise en cuvre de la rupture est un élément constitutif du processus d'écriture. J'ai eu l'occasion d'en souligner les paramètres majeurs. Le déplacement en est sans aucun doute la trame principale. Plus que l'exil, il faudrait évoquer ici la pérégrination, cette quête menée de l'Irlande à Paris, de Kassel à Londres; quête menée dans l'épuisement de ne pas avoir trouvé corps pour l'œuvre à venir. 
La rupture apparaît chez ces deux sujets comme la brutale actualisation d'un départ qui condamne à une forme extrême de dessaisissement. De façon significative, Beckett et Bion écriront des cuvres dont l'enjeu central est la perte d'identité. C'est le cas chez Beckett d'une écriture déambulatoire, acharnée à trouver une signification qui se dérobe sans cesse. Quant à Bion, son travail psychanalytique l'amènera à soumettre des réflexions complexes sur la déliaison de l'appareil psychique chez le sujet psychotique. Cette perte d'identité, qui n'est pas étrangère à l'acte de quitter sa terre natale, on la retrouve aussi chez Bion et Beckett. Cette perte d'identité loge bien sûr un facteur traumatique.

Mais ne négligeons pas pour autant l'euphorie de la création en une terre étrangère. Beckett quitte l'Irlande pour la France. Bion quittera l'Angleterre pour les États-Unis. On jugera peut-être inutile cette comparaison de deux parcours qui ont pour caractéristique première de révêler la singularité de déplacements qui prennent place dans des contextes fort différents. Le jeune Beckett quitte l'Irlande afin de devenir un sujet possédé par la passion de la littérature. Il est happé par cette puissance du voyage qui est aussi l'affirmation d'un désir d'écriture. Pour Beckett, il s'agit tout autant de devenir écrivain que de mériter la reconnaissance qu'octroie un titre qui ne se monnaye pas. Il s'agit en somme de devenir écrivain afin d'être sujet. Quitte à abandonner la défroque du sujet afin d'accepter cette perte d'identité qui condamne l'écrivain à ne plus savoir à quel monde il appartient.

Le parcours de Bion est différent, mais coïncide néanmoins avec un projet d'écriture dont on peut penser qu'il vivait de façon souterraine dans le corps de l'œuvre théorique. Bion est ce psychanalyste émérite, bardé d'honneurs, qui quitte Londres à l'âge de soixante-dix ans afin de plonger dans le tumulte californien de la fin des années soixante. Cette rupture n'est pas anecdotique. On ne peut qu'être frappé, en somme désarçonné par ce qui donne l'aspect au tout premier abord d'un ridicule de situation. Que vient faire le vieux Bion à Los Angeles? Notre surprise ne vient-elle pas du caractère stimulant de ce déplacement dans la mesure où il témoigne d'une disponibilité psychique au remaniement de représentants inconscients? 


\section{La psychanalyse : un voyage au long cours?}

J'aimerais évoquer à cet égard la pertinence d'un travail de traduction qui permet d'aménager un espace potentiel ${ }^{4}$ afin que le sujet puisse s'y déployer sans contraintes ni inhibitions majeures. Bion et Beckett furent en somme des " voyageurs potentiels ". Soudés à leur table d'écriture, sans compter pour l'un la nécessité quotidienne du fauteuil d'écoute, ils demeurèrent sensibles à cette pérégrination imaginaire que représente le travail de la Lettre.

Bion quitta l'Angleterre et devint pour l'institution psychanalytique américaine un personnage embarrassant qui donnait l'image d'un étrange personnage découvrant une terra incognita. Que venait faire Bion en terre étrangère : imposer sa pensée, diffuser le renouvellement de la théorie kleinienne ou, pire encore, révéler une ceuvre hybride nourrie de mathématiques, de philosophie et de littérature? La question peut être posée d'une autre façon : quelle étrange quête animait le séjour de Bion? Nous le savons partiellement aujourd'hui par les écrits qui ont parsemé ce séjour californien. De nombreux séminaires en Californie et en Amérique Latine, l'écriture du Memoir, une activité soutenue de psychanalyste et de conférencier. Faut-il s'étonner que l'écriture du Memoir ait coïncidé avec cette pérégrination?

Peut-on avancer que l'écriture fut associée chez Beckett et Bion à la figure du voyageur potentiel? Beckett se situe comme un auteur en rupture de ban, à la recherche d'une source créatrice qui témoigne par ailleurs de la valeur attribuée à l'exil. Il faut quitter la terre-mère afin d'écrire, tel est ce leitmotiv qui parcourt l'œuvre de Beckett et qui obéit à cette emprise du matricide joué dans la littérature. Ce signifiant du meurtre maternel permet par ailleurs au sujet de penser soulager la problématique cdipienne et la triangulation qu'elle instaure : rompre avec "l'origine " matemelle suscite la revendication d'une unité dont il s'agira de témoigner envers et contre tous.

On peut évoquer à cet égard la permanence d'un corps à corps avec la mère qui définit la configuration odipienne. Cette relation est la

'D.W. Winnicott, Playing and Reality, New York, Basic Books, 1971. 
source d'une proximité qui laisse le tiers paternel dans l'ombre. Façon de dire que le refoulement est déjà à l'ceuvre dans cette version de l'CEdipe que nous retrouvons ici et que le père, présenté comme un protagoniste discret, est à sa manière un personnage censuré à l'occasion de cette mise en scène du désir.

A insi, la mère détestée, aimée et ravie est le porte-parole d'une unité qu'il s'agit de détruire pour mieux en attester la permanence sur le front ædipien. Cette relation atteste, seion d'autres modalités, la prédominance d'un jumeau imaginaire dont nous avons relevé l'importance dans la pensée de Bion et, plus encore, dans l'œuvre autofictionnelle de Didier Anzieu ${ }^{5}$ ' intéressant aux correspondances de pensée chez Beckett et Bion.

Il faut savoir que l'œuvre théorique du jeune Wilfred R. Bion prend son envol en 1950 avec la présentation à la Société britannique de psychanalyse d'une communication sur le « jumeau imaginaire ". Le jumeau imaginaire instaure la figuration d'un double archaïque; il permet aussi de faire jouer les caractéristiques de l'identification projective en proposant a l'image $n$ d'une psyché altérée. En somme, le jumeau imaginaire fait intervenir dans la pensée de Bion cette évocation du presque-semblable, qui rappelle la figure du prochain mise en exergue par Freud. Le jumeau imaginaire est d'abord pour Bion cette altérité forte, par ailleurs élaborée grâce au recours à la mêmeté, qui permet au sujet de se reconnaître à la fois semblable et différent. Bion propose d'ailleurs la genèse de ce jumeau imaginaire : a [...] [il] remonte à sa relation la plus précoce et traduit son incapacité de tolérer un objet qui ne soit pas entièrement sous son contrôle. Le jumeau imaginaire avait donc pour fonction de dénier une réalité autre que lui ${ }^{6} "$. Bion fait référence au jumeau imaginaire afin d'identifier le rôle joué par les mécanismes d'introjection, de projection, de clivage, de personnification dans le cadre analytique. Il fait appel à l'analyse des rêves, à l'étude des associations libres afin d'indiquer l'importance de ce motif clinique. Bion rend compte

${ }^{5}$ Didier Anzieu, Beckett et le psychanalyste, Paris, Mentha-Archimbaud, 1992.

6 W. R. Bion, " Le jumeau imaginaire ", dans Réflexion faite, Paris, Presses Universitaires de France, coll, * Bibliothèque de psychanalyse w, 1983, p. 25. 
du déroulement de trois analyses qui présentent une isotopie de la gémellité. Selon Bion, chaque patient personnifie a contrario des parties clivées de sa personnalité. La majeute partie de l'étude est consacrée au seul patient ayant pu créer un jumeau entièrement imaginaire ( $\alpha$ A $x$; toujours selon Bion, les deux autres patients ont recours à des mécanismes schizoïdes qui sont amplifiés par une véritable gémellité dans leur cercle familial ou social. Bion propose dans cet article novateur une réflexion sur l'oscillation qui caractérise le passage de la position dépressive à la position schizo-paranoïde; il note à cet égard la ténacité de ces mécanismes qui parasitent la relation thérapeutique : l'analyste tient lieu de jumeau protéiforme jusqu'à ce que le patient soit en mesure d'intégrer des objets totaux (internes) et de faire la synthèse de processus psychiques liés au développement des organes des sens.

La figure du " jumeau imaginaire " recourt aux motifs structurants de la névrose obsessionnelle, mais aussi de la paranoïa puisque le sujet se reconnaît dans la démesure de la certitude que lui octroie le regard présumé envieux d'autrui. Le jumeau tient lieu d'ombre silencieuse; il incarne de plus la dimension dépersonnalisante, ou quasi démoniaque, qui fait de l'autre-en-soi la fondation d'une identité archalque refoulée.

C'est parce que l'interlocuteur maternel affiche sa toutepuissance qu'il est vital de modifier, d'amoindrir la violence interprétative dont elle est le porte-parole afin de retrouver la forme atténuée de cette imago sous l'aspect du jumeau imaginaire. Si l'on retient cette perspective de travail, Beckett fut un répondant de la pensée de Bion. On pourrait avancer que Beckett fit de la littérature ce jumeau qui permettait de matérialiser une survie favorisée par le passage à la langue étrangère. En somme, le souhait matricide était si puissant chez Beckett qu'il nécessitait un abandon des signifiants voués à la langue première. La littérature représentait peut-être alors cette forme majeure d'accompagnement dans la solitude inventoriée par ce jumeau imaginaire. Quant à Bion, l'on sait qu'il consacra un segment de son cuvre à la transmission du trauma que figure ce jumeau imaginaire : représentation qui est à la fois projection schizoïde et mise en valeur d'un secret. La question de la traduction mérite d'être posée de nouveau à partir de cette figure du déplacement qui laisse percevoir le fondement créateur du trauma. 
La figure du jumeau imaginaire requiert encore une fois notre attention. À la fois projection schizoïde d'une partie clivée de la personnalité et source d'un enveloppement protecteur, le a jumeau imaginaire $»$ est l'expression même de la sédentarité. Faut-il s'étonner que Didier Anzieu retrouve dans l'ceuvre de Beckett la persistance de cette représentation? A l'abandon de la figure maternelle et des signifiants associés à la mise en valeur de la terre natale, ne fallait-il pas pour Beckett opposer ce sentiment d'identité primaire qu'incarne un double à la fois proche et lointain?

Ainsi Beckett laisse en jachère la langue anglaise afin de mieux explorer la signification d'un matricide linguistique qui se traduit par l'acte de quitter l'Irlande. Chez Beckett, l'acte d'écrire suppose une renonciation majeure, l'abandon de la psyché maternelle dont il faudra traduire les signifiants dérivés dans une matrice linguistique dont la valeur est éprouvée à la faveur de la distance. Beckett aura été ce voyageur de * Ialangue ", semblable aux personnages tragiques qui accompagnent la genèse des grands mythes à partir desquels la psychanalyse structure son propre champ symbolique. Le parcours de Beckett impose de renoncer au pays immémorial de la mère. Ce parcours est aussi cette injonction silencieuse du père qui interdit de partager les signifiants amoureux liés à la possession de la langue matemelle.

La signification majeure du corps à corps peut être étayée grâce à ce constat. L'injonction silencieuse du père n'est pas l'aveu d'une défaite, mais plutôt un repli stratégique. Si le père ne s'impose pas, c'est qu'il connaît la force de ce silence qui marque le sujet de façon permanente. Le corps à corps avec la psyché maternelle est favorisé par la démesure de ce silence du père qui est à sa manière un signifiant de démarcation. La mère et le fils, en témoigne Beckett, peuvent bien lutter amoureusement afin de revendiquer la possession d'un signifiant premier, il reste que cette quête de la langue-mère condamne à l'exil. Ce qui justifie la permanence des interdits adipiens : le père est un rival qui ne saurait s'accommoder de la figure mimétique incamée par le jumeau imaginaire. Faut-il s'étonner dès lors que Beckett ait pris ses distances avec la mère-patrie afin de créer dans une autre langue, ce qui lui permettait de contrer l'impact psychosomatique destructeur d'une coincidence de pensée avec la mère? Chez Beckett, la sanction du départ rappelle cette volonté de renaître sous un autre jour. 
La littérature permet de s'offrir le privilège de devenir un objet de traduction. Face à l'omnipotence des signifiants associés à la languemère, le sujet instaure l'œuvre à créer grâce à la mise en valeur d'un fantasme d'auto-engendrement. Devenir soi dans une langue autre, tel est l'enjeu déclaré. La démesure de la tâche est à situer dans une perspective où le narcissisme apparaît un puissant facteur d'identification. La litterature cristallise cet enjeu puisqu'elle offre un contenant de pensée où le sujet peut s'éprouver dans une langue " autre " qui est malgré tout fidèle à soi.

Pour Beckett, l'acte de renaître dans la langue française permet de nommer latéralement la proximité incestueuse nouée avec la langue maternelle. Le psychanalyste face à ce projet se trouve lui aussi en terrain familier. Le psychanalyste travaille avec les matériaux offerts par la langue-mère. Il se doit cependant, c'est sans doute ce qui le différencie de l'écrivain, de ne pas laisser place à l'emprise passionnelle qui l'amènerait à déclarer l'amour de sa langue. Que l'on me comprenne bien. Je n'affirme pas que le psychanalyste est le sujet neutre et objectif d'une métacommunication. L'inconscient est plurilingue et n'accepte pas d'être associé au génie de la langue. C'est pourquoi le psychanalyste se doit en ce domaine de rester circonspect. Le psychanalyste est l'enfant de $s a$ langue maternelle. À ce titre, le psychanalyste appartient à sa culture et ne saurait en aucun cas la rejeter. La préhistoire infantile, tout comme la mémoire culturelle, contribuent a former ce que sera tel sujet. Le psychanalyste peut bien avoir son style à l'oral et à l'écrit. Dans cette perspective, il ne se différencie pas de l'écrivain qui forge sa signature afin de s'octroyer un Nom propre. Par contre, le psychanalyste ne peut ignorer que la mémoire culturelle de la langue maternelle appartient à un univers restreint dont il est l'agent. Or je le rappelle : l'inconscient est plurilingue. Une analyse peut être menée en français, en anglais, ou encore en italien. L'aire culturelle, les codes sociaux, les aménagements de la configuration odipienne, tous ces facteurs, parmi bien d'autres, nuancent, on en conviendra, l'universalité de la cure-type. Mais l'analyse demeure, malgré les formes diverses d'introspection proposées dans un monde culturel hybride et contingent, la capacité renouvelée d'écouter la parole de l'Autre. Voilà encore une fois pour quelles raisons le psychanalyste se doit de demeurer circonspect. Sa langue maternelle, it la parle certes. On peut aussi avancer qu'il la partage avec un patient qui y verra matière à une première intimité endopsychique. Mais le 
psychanalyste ne doit pas oublier qu'il ne s'identifie pas par sa langue maternelle, qu'elle ne constitue pas l'instrument naturel d'une communication où l'inconscient aurait sa place.

En témoigne le titre de l'ouvrage de Didier Anzieu, Beckett et le psychanalyste. Si Beckett peut être nommé, c'est qu'il obtient grâce à l'écriture la sanction d'un nom propre qui le signe comme sujet. Il en va autrement \& du psychanalyste » dont la solitude et le souci de discrétion sont choses essentielles. Bion aura été à cet égard un personnage fascinant. D'une extrême rigueur en ce qui a trait à l'exercice du métier de psychanalyste, soucieux d'abolir la mémoire et le désir au coeur de l'écoute du patient, Bion accepta de plonger dans le monde de la fiction. L'écriture du Memoir, à l'occasion du périple californien, rend compte de cette ouverture à la littérature à travers la multiplicité des discours inventoriés.

La facture de la trilogie autofictionnelle $A$ Memoir of the Future est singulière. Bion compose un portrait de groupe sur fond de catastrophe. Alors que la menace diffuse d'un envahisseur - réminiscence de la guerre de 1914-1918 où Bion fut sous-lieutenant pèse sur une communauté en proie à une profonde turbulence émotionnelle, $A$ Memoir propose un discours polyphonique où chaque protagoniste tente de donner voix à l'inconscient. Ce livre tente donc de saisir, par le recours à une multitude d'énonciateurs, la complexité de la parole humaine. Les protagonistes sont soumis à un huis clos angoissant puisqu'ils interprètent devant nous un dialogue qui inscrit à même la chair des interlocuteurs une turbulence émotionnelle qui amplifie le sentiment de terreur vêcu par l'envahissement.

Le propos de Bion est radical. La turbulence émotionnelle est le signe d'une déliaison de la structure narrative. Bion ne se contente pas d'instaurer un parloir dialogique où chaque interlocuteur aurait droit à la parole. Le dialogue ne favorise pas la pleine et entière connaissance de soi. On ne retrouve pas á cette occasion la lente progression du a travail s de la pensée, travail engagé par le nécessaire ajustement de proximité que permet de faire jouer le dialogue. Bion tente plutôt de faire apparaître la mise en cuvre de l'inconscient qui fracture le lien de pensée. Cette mise au jour résiste à l'enfermement diégétique qui condamne la parole à 
devenir un récit. Ce n'est d'ailleurs pas un hasard si Bion recourt dans son travail d'écriture à la forme autofictionnelle.

Le déplacement de la " voix " narrative est manifeste. Bion ne témoigne pas de " soi " à la faveur de l'exercice autobiographique. L'écriture du Memoir inscrit la fragile mais nécessaire destitution du sujet qui accepte de s'entendre penser comme s'il était un autre. À vrai dire, l'usage de la comparaison est ici inutile : il ne s'agit pas de s'entendre penser comme un autre mais de devenir, et ce littéralement, un autre-quesoi à la faveur de cette perdition que crée la littérature. On s'étonnera à juste titre du caractère extrême d'une telle expression : se perdre, n'est-ce pas là une injonction dont la passivité feinte conteste le protocole autobiographique?

Philippe Lejeune fait valoir sur ce point la nécessité d'une identité de reconnaissance qui se construit à partir de la conjugaison des figures de l'auteur, du narrateur et du protagoniste. Reconnaissons qu'il ne s'agit pas ici de se perdre, mais tout au contraire d'assurer le magistral contrôle de soi à la faveur d'une corrélation des registres référentiels et fictifs. Se perdre par le biais de l'acte d'écriture, n'est-ce pas d'une certaine manière retrouver ce qui fonde l'acte analytique? S'entendre parler révèle-t-il, du côté de l'analysant, cette illusion d'une voix narrative qui se donne à entendre comme pleine et entière connaissance de soi?

Se perdre dans l'acte de parole, c'est accepter ce * il n impersonnel qui appartient de plein droit, selon Maurice Blanchot, à la voix narrative. Ajoutons que Bion tenta par l'écriture de donner un corps et une voix à cette passivité de l'écoute qui accepte de rompre avec l'illusion de la durée. La psychanalyse, si elle tente de faire corps avec l'inconscient, ne peut mimer le rythme et les altérations imposés à l'appareil psychique. La psychanalyse se doit, à cet égard, de faire appel à la narration afin de créer un espace de parole qui permet la mise en œuvre du dialogue. Ce dernier, on l'a vu, peut caractériser une parole sûre d'elle-méme, assertive, arrogante. Mais la psychanalyse sait que le discours est la forme rationnelle de la parole qui paie secrètement tribut au processus primaire. C'est à travers les lapsus, les jeux de mots, les apparents contresens que la psychanalyse octroie à la narration son statut de traducteur. J'entends par là que la narration n'est pas la forme de l'inconscient : elle en est l'imparfaite figuration. Ce qui ne signifie pas 
que cette imperfection est la marque tenace d'une impureté qui corrompt l'inconscient, cette luminosité qui échappe, par l'indicible proclamé, à toute forme de mise en discours. La narration est l'artifice nécessaire de la psychanalyse. Sans narration, le sujet serait en proie aux affres du début et de la fin du monde.

Et l'analyse, dans la mesure où elle contribue à instaurer un cadre de séance, n'échappe pas à cette dure réalité du récit qui impose de mouler le sujet dans une narration qui le vêt. L'acte de traduire est peutêtre le motif premier de la rencontre analytique. Le sujet qui se présente au cabinet de l'analyste demande d'une certaine manière à être entendu pour ce qu'il n'est pas. À moins qu'il cherche difficilement à déclamer ce qu'il a été.

\section{Devenir un autre-que-soi...}

Voilà pour quelles raisons la rencontre de Samuel Beckett et Wilfred $R$. Bion a pour nous une résonance souterraine sur cette question de la traduction. Beckett ne se présenta pas devant Bion sous la forme de l'illustre écrivain en quête de reconnaissance. 11 y avait de l'humilité et de la souffrance dans ce désir d'aller dire de sa personne les infortunes du mal-être. A cette occasion, Beckett sut devenir un autre-que-soi. Beckett n'était pas seulement un sujet qui doutait de soi, hésitait à propos de son désir d'écrivain. Beckett ne se présenta pas devant Bion en faisant jouer le miroir de l'imposture, mentant de belle façon sur ce qu'il prétendait être, ou encore sur ce qu'il avait été. Il y a de l'imposture, qui est la force cachée de la sincérité, lorsque le sujet instaure l'identité comme cette polarité essentielle à partir de laquelle il fonde son existence. Beckett était véritablement un autre-que-soi lorsqu'il rencontra Bion. Il lui fallait le truchement d'une parole étrangère afin que la traduction prenne son envol et que le sujet-analysant soit interprété dans l'incompréhension qu'il suscitait.

On s'étonnera peut-être de la torsion que je fais subir au destin de l'analyse. Cette demière n'est pas la reconnaissance progressive d'une inquiétante familiarité qui demande à être patiemment incanée. S'il y a au cœur de l'analyse ce passage du temps qui est l'objet de la perlaboration, le travail mis en cuvre n'obeit pas uniquement à ce présupposé canonique d'une identité narrative qui saisirait à bras le corps 
l'inconscient. $\mathrm{ll}$ faut aussi savoir échapper à cette identité narrative qui suppose l'altération du sujet par la durée dont il est l'agent. Il faut savoir reconnaître que la psychanalyse est l'agent radical de traduction d'une altérité qui échappe à tout désir de contrôle narratif. La turbulence émotionnelle que revendique Bion appartient à ce monde de l'infigurable. Au contraire de l'indicible qui affirme l'impossible à dire comme source même de la parole, l'infigurable rend compte d'un autre processus.

Il nous faut revenir à cette turbulence émotionnelle, revendiquée avec force par Bion, afin de mieux saisir ce que l'inconscient emprunte à ce chemin de traverse. Si l'indicible qualifie un manque-à-dire dont il est espéré - faussement - la résolution, l'infigurable est ce travail avec la matière corporelle qui permet d'investir la pensée. L'analysant, lorsqu'il s'engage dans la cure, sait qu'il y a un point de non-retour. Après avoir épuisé les balises dites autobiographiques du récit de soi, l'analysant perçoit, et c'est là une faille majeure dans la construction d'un ordre narratif, que le manque-à-dire appartient au monde onirique de l'infigurable. Cette faille majeure, qui entrave l'énoncé et fait surgir le désir d'être-un-autre au cceur de l'acte de parole, n'a rien à voir avec les vertiges de l'indicible. L'analysant n'est pas à la poursuite d'une vérité énigmatique dont le scintillement demanderait à être saisi sous la forme d'un signifiant.

La figure que je viens de décrire donne l'illusion d'une captation autobiographique que l'énoncé de l'analysant permettrait de concrétiser. Or l'analysant est à la recherche d'un tout autre savoir. S'il y a bien une quête animant la parole de l'analysant, cette demière n'appartient pas au registre mimétique de la connaissance de soi. Ceux qui sont passés par "l'expérience " de l'analyse savent que le savoir dont il est question n'a rien à voir avec l'approfondissement de la connaissance de soi, le mûrissement de la personnalité, l'acceptation pleine et entière des défauts et qualités qui composent le portrait du sujet parlant. Ce qu'entend la psychanalyse en ce domaine est radicalement différent : l'expérience s'acquiert par la mise en valeur de l'oubli qui permet de se savoir autreque-soi. Ce qui nécessite en retour, aussi bien pour l'analysant que pour l'analyste, cet appel de la traduction.

Faut-il s'étonner que Bion, lors de l'écriture du Memoir, se soit soumis, par le biais de la fiction, à pareil exercice? Au contraire de 
l'autobiographie qui fait appel au vertige de l'indicible, l'étrange trilogie autofictionnelle que nous propose Bion dans $A$ Memoir of the Future est cette mise en jeu de l'infigurable. L'autobiographie requiert en effet la transparence et la luminosité : conditions essentielles afin que le sujet puisse se dévoiler et ainsi offrir les conditions d'une pleine et entière connaissance de soi. L'identité habite le protocole autobiographique et le sujet qui tente de proposer une traduction de soi se doit de nier qu'il y a fondamentalement ce jeu de l'infigurable au cour du discours. Le passage à l'écrit que fait valoir Bion lors de son périple californien peut être entendu comme ce désir d'abandonner la somme autobiographique afin de devenir un autre-que-soi. L'autofiction permet d'en finir avec l'insistance identitaire et de transformer l'Auteur en personnage parodié, travesti, déployé au gré des circonstances sous de nouvelles vêtures.

On sait que l'autofiction, concept proposé par Serge Doubrovsky afin de définir la portée d'un genre littéraire dont il se déclarait l'initiateur', fait jouer, dans le cadre de l'énonciation autobiographique, un renversement de perspective assez troublant. Serge Doubrovsky se plaît, c'est le moins qu'on puisse dire, à mêler les cartes. L'autobiographie n'est plus le signe d'une vérité révélée, encore moins l'aveu prosaïque d'une sincérité à l'œuvre. L'autobiographie devient pour Doubrovsky la mise au jour d'un jeu avec le signifiant de l'identité. Il n'est plus question ici d'une incarnation de l'identité que l'écriture aurait charge de transmettre. Pour les mêmes raisons, la fiction n'est pas le libre exercice d'une volonté créatrice. La fiction, qui apparaît comme l'incarnation du génie créateur, est radicalement bouleversée par l'intrusion d'un discours qui accepte de se définir comme un univers contingent, soumis à la puissance du récit. Devenir autre-que-soi par le recours à ce singulier métissage qu'inaugure l'autofiction, n'est-ce pas justement accepter d'être objet de traduction?

En somme, Bion, écrivant $A$ Memoir of the Future, acceptait peut-être de devenir ce sujet pensé par une fiction qui l'habitait. L'écriture devenait un acte intime, particulièrement sensible à ce que la psychanalyse peut faire entendre de résonances. Si l'analyse se révèle

${ }^{7}$ Serge Doubrovsky, “ L'initiative aux maux : écrire sa psychanalyse ", dans Parcours critique, Paris, Éditions Galilée, 1980. 
dans le défaut des langues, c'est parce qu'elle reconnaît le caractère plurilingue de l'inconscient. Prétendre parler sa langue maternelle, c'est bien sûr affirmer un fort sentiment d'appartenance. Mais cette heureuse saisie de la langue matemelle est un moment fugace qui appartient au règne de l'éphémère, destinée dont Freud soulignait la pertinence culturelle. Moment fugace en effet que cette heureuse possession de la langue première. Le psychanalyste sait en ce domaine que les illusions sont de courte durée. Traduire est cette tâche patiemment poursuivie qui noue la relation dialogique que partagent l'analysant et l'analyste. Si l'inconscient est plurilingue, c'est parce qu'il se caractérise par une mobilité énonciative qui recourt à l'ordinaire du quotidien.

C'est pourquoi Bion écrivit $A$ Memoir of the Future. Acceptant de $s^{\prime}$ inscrire au cour du protocole autofictionnel, Bion devenait un sujet écrit par une cuvre en cours. 11 acceptait somme toute de devenir un protagoniste d'encre et de papier. Fidèle en cela à la trame théorique qui parcourt son ceuvre, Bion acceptait de devenir, grâce à l'instauration salutaire de la fiction, une "pensée vide "accueillant les représentations du monde inconscient. L'œuvre en cours, qui n'est pas sans relations avec la somme joycienne, inscrivait une pulsion traduisante, soucieuse de faire entendre les voix multiples de l'inconscient. La traduction, dès lors qu'on accepte d'entendre la singularité qu'elle revêt en psychanalyse, n'est pas autre chose que cet exercice tenace d'altérité.

Beckett fut entendu par Bion avec ce désir de devenir un autreque-soi. J'insiste sur la dimension projective de ce jeu que représente la mise en scène de l'altérité. Beckett ne tentait pas, à la faveur de la cure, d'être un " autre ", de mettre fin au solipsisme identitaire par la revendication éperdue et magnifiée de l'altérité. La cure ne correspond pas à cet objectif. Il serait naîf de croire que la banalité autobiographique du sujet cède la place à l'émerveillement d'un sujet se découvrant un jour autre-que-soi. La psychanalyse est toute entière concernée par un travail de la traduction puisqu'il s'agit bien ici de faire entendre par le langage un processus où l'inconscient se donne à lire dans la démesure de ses effets. Beckett, comme le patient anonyme qui s'allonge sur la couche de transferts de l'analyste, est un sujet qui énonce la dimension agentive de l'altérité. 
L'analysant n'est pas l'icône de l'altérité. Parce que le travail de la traduction fait appel dans le domaine psychanalytique à l'infigurable, le sujet-analysant sait que la parole dans la cure est incomplète, partielle, fragmentée. Il ne saurait être question sur ce point de revendiquer une authenticité fondatrice, une anamnèse dont l'efficacité serait si redoutable qu'elle amènerait le sujet à se percevoir tel qu'en lui-même. Cette agentivité de la cure est donc le fruit d'un processus, d'un * devenir n qui prend la forme d'une action différée.

C'est bien le sens que Freud octroie dans ses écrits à l'expression Nachträglichkeit. Cette a action différée " est aussi traduite en français par l'après coup. Dans les deux cas, l'apport freudien est majeur. Il n'est plus question de la reconstitution de la mémoire infantile, mais plutôt d'un travail inconscient qui donne toute sa place à la reconstruction agentive des signifiants majeurs de l'histoire de l'analysant. La notion d'action différée, telle que Freud nous en propose la définition, est déterminante puisque le sujet ne s'y voit pas désigné comme le seul responsable de ses actes de parole. Faire référence à $\alpha$ l'action différée $n$, ou encore à $\alpha$ l'après coup ", c'est indiquer qu'il y a, au cour de l'expérience analytique, une surdétermination inconsciente qui fait appel à la traduction. Si le fait de devenir-un-autre qualifie un processus, c'est à la condition d'indiquer que le sujet se traduit comme altérité pensante dans le cadre de la cure.

Il en va de même de l'acte d'écriture, tel que je le conçois aujourd'hui. L'autofiction bionienne peut représenter à cet égard un témoignage exemplaire. L'écrivain-psychanalyste n'est pas ici le Maître d'une parole qui demande à être incamée sous forme d'ecrits. L'écrivainpsychanalyste est plutôt convoqué à la place qu'identifie cette a pensée vide * : a priori, dont la source kantienne chez Bion n'est pas négligeable, et qui permet à l'écrivain de devenir un autre-que-soi. On avancera dès lors que l'écriture et l'acte de parole dans la cure peuvent être conjugués puisque le sujet s'y perçoit réfractaire à toute intention manifeste, à toute déclaration formelle.

Les conséquences d'une telle perception de l'acte analytique sont majeures. Je veux ici saisir in vivo la singularité de la parole psychanalytique comme si le défaut de traduction pouvait permettre le recours au langage. Le psychanalyste, s'il est d'abord un " voyageur potentiel n, est aussi un voyageur immobile. Il circule dans le monde des 
signes - sous cet angle il partage les mêmes préoccupations que l'écrivain - tout en sachant qu'il est soumis à une obligation de réserve. Cette obligation est justifiée par la nécessité de soutenir - et maintenir un silence dont la parole est l'accompagnant. Cette parole, on l'aura deviné, appartient à lanalysant. Pour qu'il y ait traduction, l'analyste doit donner corps aux mots. Plus encore, il doit occuper cette place discrète qui lui impose d'être un passeur de mots, de voyager dans l'arène du langage tout en permettant à l'analysant de se déplacer et de créer, par le remaniement des signifiants qu'il joue dans la cure, cette traduction qui l'institue sujet.

Cette exigence n'est pas le fruit d'une déclaration. Elle n'appartient pas aux conditions dites préalables de la cure (l'associationlibre, le nombre de séances, les modalités de paiement). Traduire ne s'impose pas, surtout lorsqu'il s'agit de saisir à l'intérieur de sa propre langue un signifiant inédit qui frappe par son caractère radical et hétérogène. Le psychanalyste partage avec l'écrivain cette passion pour les mots. Il partage aussi sans doute avec l'écrivain cette passion nouée avec le silence des mots - ce qui appartient de plein droit à l'exercice de la traduction. Il faudrait alors entrevoir le passage à l'écriture chez le psychanalyste comme cette ouverture à la traduction de l'idiome psychique.

L'aventure littéraire de Bion accompagne cette avancée du langage. L'écriture du Memoir est un acte de dramatisation qui amène le psychanalyste aux côtés d'CEdipe afin de faire surgir, par l'épreuve même que le langage instaure, cette puissance de la transgression. Écrire permet au psychanalyste de traduire dans sa propre langue les idiomes transférentiels qu'il écoute. En somme, l'acte d'écrire permet de retrouver dans la destination de l'ouvre la figuration d'un analysant rappelant le « jumeau imaginaire » qui inaugure l'ouvre bionienne.

\section{Le rêve de Babel}

Le parcours que je retrace n'est pas éloigné de la trame transférentielle qui inaugure la rencontre entre Bion et Beckett. Le premier fera ceuvre de psychanalyste. Il se soumettra avec rigueur à l'exigence pulsionnelle d'écouter le matériau inconscient pour en faire surgir la résonance secrète. Mais cette résonance, Bion tenta aussi de la faire entendre sous forme 
d'écrits. La source première de l'écriture fut pour Bion la théorie, la glose métapsychologique. Mais peu à peu cette matrice théoricienne fut abandonnée au profit d'une déliaison mémorielle que favorisait l'écriture. Il semble que le registre autofictionnel pratiqué dans le Memoir ait permis d'aménager un espace de jeu entre l'exigence de parler de l'expérience analytique tout en sachant que cette dernière est soumise à un * travail de la traduction * qu'instaure la littérature.

La réflexion menée par Bion dans le monde de la théorie est d'une précision extrême. Pour seuls exemples, indiquons son intérêt pour la géométrie, les mathématiques, la logique. Un des rêves théoriques de Bion fut sans doute d'extraire la psychanalyse du langage illustratif de la métapsychologie. Bien que cela traduise un tout autre champ de réflexion, on doit souligner la pertinence scientifique de l'écriture de Bion, sa volonté de saisir avec rigueur et discernement ce que la psychanalyse emprunte aux avancées d'autres lieux du savoir. La passion mathématique de Bion rejoint d'une certaine manière l'amour de la littérature. Pour Bion, l'apport des mathématiques, mais tout aussi bien de la géométrie non euclidienne, est majeur puisque le réel peut être saisi sans qu'intervienne la nécessaire corruption qui entrave l'énonciation du sujet parlant. Ce rêve, on aura l'occasion d'en mesurer la portée, est d'une certaine façon le désir affirmé de promouvoir une pensée qui échappe au temps de Babel. Mais parce que le discours scientifique étudie les traces infra-signifiantes du réel, il appartient au monde du clivage, de la dispersion, somme toute de la traduction. Ce qui explique peut-être l'ambivalence apparente de la pensée de Bion à ce sujet. Le domaine scientifique - je pense notamment à la fameuse mathématisation de l'expérience analytique avec * The Grid * - traduit ce désir de transparence conceptuelle. Comme si la psychanalyse se devait de faire appel à un invariant mathématique qui puisse construire une esquisse du réel échappant aux contraintes du malentendu.

Pourtant, la psychanalyse ne cesse d'écouter ce malentendu. La parole du sujet est tâtonnante et tente difficilement d'en arriver à une certitude qui aurait valeur de topique. Est-ce pour cette raison que Bion fit appel à cette autre expérience qu'est la littérature? Nous avons eu l'occasion de faire valoir que le " travail de la traduction " inaugure un abandon de soi dans la mesure où le sujet accepte d'être parlé par les divers idiomes qui composent son monde psychique. Ce monde, il faut en 
convenir, ne possède pas de cohérence interne. Traduire en son for intérieur la langue maternelle qui nous situe comme sujet, c'est accepter cet autre-que-soi dont j'ai eu l'occasion de préciser la structure.

Avançons par ailleurs que l'écriture fictionnelle inaugure un salutaire laisser-aller que Bion accepta avec plaisir. Si Beckett abandonna I'Irlande pour poursuivre son ceuvre d'écrivain, on peut avancer de façon un peu provocante que Bion abandonna la psychanalyse afin de devenir un homme de lettres. Le désir matricide accompagne l'émergence de l'ouvre de Beckett. Il s'agit en effet d'écrire dans une langue seconde afin d'autoriser la sublimation d'un corps à corps meurtrier avec l'imago maternelle. Chez Bion l'acte d'écriture du Memoir prend la forme d'un sémanticide. Bion tente par cet écrit de mettre à mort le langage cuirassé, doxologique de la parole psychanalytique afin de permettre la venue de la Chose en soi. Cette source kantienne qui habite l'wuvre theorique et fictionnelle de Bion suppose que l'a priori inconscient (ce que Bion nomme la préconception) trouve matière à penser sous la forme d'une conception qui permet l'avènement d'une réalisation émotionnelle. L'écriture du Memoir est pour cette raison d'une grande richesse. Elle donne voix à l'émergence d'une parole qui tente de traduire l'inconscient.

Cette parole-écriture est enfin liée à quelques segments des travaux de Bion sur la structure mythique. Bion consacrera certains de ses écrits à la figure du mythe de Babel'. Chez Bion, le mythe de Babel est la source de la pulsion épistémophilique. Le désir de connaître - l'appétit sexuel que fonde la curiosité - introduit dans le champ du langage la représentation symbolique de la sexualité orale et de la dispersion linguistique.

Le désir d'une langue commune - affirmation suprême de toutepuissance qui concurrence l'omnipotence de la parole divine - traduit une curiosité sexuelle qui fait appel à l'oralité. La destruction de Babel signifie par ailleurs que le langage est la seule demeure de l'humain. C'est à la faveur de cette dispersion " fondatrice * de la communauté des

\footnotetext{
'Wilfred R. Bion, Recherches sur les petits groupes, Paris, Presses Universitaires de France, coll "Bibliothèque de psychanalyse ", 1965; Eléments de psychanalyse, Paris, Presses Universitaires de France, coll. * Bibliothèque de psychanalyse $*, 1979$.
} 
hommes que le sujet trouve son chemin dans le monde du langage. Selon Bion, l'Après-Babel fait du langage un lien de pensée dont la dimension symbolique est pleinement mise en valeur. Il s'agit de connaître le monde - et de fonder la connaissance de soi - à partir de cette dispersion qui traduit l'abandon de la parole sacrée. En somme, Babel est le fondement de la connaissance narrative dont la psychanalyse nous offre le pourtour. C'est parce que nous sommes des sujets soumis au défaut des langues que nous tentons, bien imparfaitement, de renouer avec le désir d'un signifiant premier. La sexualité orale appartient de plein droit à ce monde de la fondation et de la dispersion.

Sans doute est-ce pour cette raison que la pulsion épistémophilique trouve dans le regard dévorant de l'enfant la source d'une première curiosité. Il en va de même de l'apprentissage des langues. Quels que soient les détours de la formation linguistique, il n'en reste pas moins clair que le savoir des langues est aussi un désir des langues. La pluralité qui est ici affichée entretient d'étroites relations avec le signifiant maternel. Et la pulsion épistémophilique est ce désir de savoir qui est étayé par la curiosité infantile. Voilà pourquoi la sexualité orale apparaît si importante aux yeux de Bion dès lors qu'il est fait mention du mythe de Babel. Façonner une langue commune, c'est interdire la source même de la traduction. Instaurer l'amour de la langue comme dépositaire de la connaissance humaine, c'est instaurer l'unité et la sacralité au coeur du processus linguistique. Bion face à ce projet demeure sceptique. La pulsion épistémophilique refuse avec une virulence peu commune l'épreuve de la frustration.

Que la parole échappe à la bouche avide qui s'est abreuvée au sein maternel, voilà en effet une séparation insupportable. Pour des raisons complémentaires, le regard ne peut échapper à la source figurative première qu'est la mère. Cette dernière n'est pas seulement une imago. Elle incarne de manière plus nette la formation archaïque d'un contenant de pensées. Si la sexualité orale est l'affirmation pulsionnelle d'une fusion avec la mère, elle indique tout autant la connaissance de soi à la faveur de l'auto-érotisme. Ne faut-il pas y voir l'une des sources inconscientes de l'amour de soi? Pour toutes ces raisons, l'amour de la langue fait appel a la traduction archaïque des signifiants associés au soma-psyché de la mère. 
L'apprentissage et la connaissance n'appartiennent pas au monde positif de la productivité langagière. Bion indique d'ailleurs l'importance du mythe de Babel dans le cadre d'une compréhension psychanalytique du langage. L'acte de connaissance nécessite le recours au langage. Mais cette connaissance consiste peut-être aussi à domestiquer la sexualité orale pour mieux faire valoir la nécessité d'une pensée génitalisée. Cette dernière appartient de plein droit au monde surmolique et à la dimension instinctuelle qu'elle institue dans le champ social. C'est dire que la psychanalyse a pour enjeu de traduire cette surcharge pulsionnelle réprimée qui a valeur de culture.

Le sujet créateur ne se contente pas de révéler ce qui lui échappe. Il n'est pas le protagoniste involontaire d'une parole qui est proférée à son insu. Il ne se situe pas dans le registre sacral d'une transmission ascendante. Le sujet de la modernité - qui est le sujet de la psychanalyse - habite le monde séculier de la parole mythique. Babel n'est pas pour les interlocuteurs que nous sommes le mythe fondateur d'une dispersion de la parole et le récit de sa possible restauration. Babel est notre mythe contemporain. La dispersion nous habite; elle est notre mémoire et notre culture. Bion aura été sensible, comme Freud d'ailleurs, à l'importance du mythe qui est une des formes discursives de la pensée. Bion ne s'intéresse pas à la facture phylogénétique du mythe qui serait le récit de l'enfance de l'humanité. Il prend ses distances avec une conception naïve et ethnocentrique de l'univers mythique pour mieux intégrer les relations complexes entre l'appareil psychique et la fabulation narrative du genre humain. Demeure cette question. Pourquoi Bion s'intéresse-t-il tant à Babel? Pourquoi y voit-il une des modalités majeures de la connaissance, sans négliger la mise en tutelle de cette connaissance face au désir inconscient?

Peut-être Bion voyait-il dans sa pratique clinique un sujet en proie au défaut de mémoire, un sujet dont la dispersion babélienne était la forme d'énonciation contemporaine? Peut-être Bion, jeune psychiatre en formation analytique à la Tavistock Clinic, rencontra-t-il chez Beckett le fantôme de cette mémoire brisée? La réflexion clinique de Bion ne cesse en effet d'affronter le vertige de l'impensable, de l'innommable. Je rappelle à cet égard que la pensée de Blanchot demeura pour Bion, sous sa forme interrogative, un enjeu de reflexion majeur. Le mythe de Babel, 
dans la pensée psychanalytique de Bion, permet de penser l'émergence de la puision épistémophilique.

L'acte de traduire, parce qu'il est ce lieu de rencontre privilégié de l'interlangue, permet d'intégrer la puision épistémophilique dans sa relation à la sexualité comme mobile pulsionnel fondamental. Pour cette raison, la traduction, dont l'un des motifs est le mythe de Babel, rencontre sur son passage, comme Bion l'indiquait, la puissance dévastatrice de l'arrogance. Quoique l'on puisse ajouter que la traduction est aussi la rencontre avec l'espace littéraire, il est naîf d'espérer que la création, perçue conme la forme achevée de l'intention littéraire, se construise à partir du stéréotype de l'inspiration.

C'est au contraire une esthétique de la disparition qui prévaut, ainsi que le suggérait dans un autre contexte Paul Virilio'. L'acte de penser la perte - c'est le discours kleinien - favorise la symbolisation de l'objet perdu. Le propos énoncé par Bion amplifie la thèse kleinienne. Pour Bion, le sujet est soumis à l'emprise catastrophique d'une pensée qui abolit l'objet, le clive et, paradoxalement, le transforme en rudiments de pensée susceptibles de donner naissance à une véritable croissance émotionnelle.

Babel demeure encore une fois le mythe de notre culture. En témoigne l'omnipotence divine qui aurait $m$ is fin de manière violente à la pulsion épistémophilique de l'enfant. En témoigne aussi l'image de l'adulte qui aurait été condamné à porter l'injonction civilisatrice du Surmoi. Babel est notre mythe contemporain. Nous vivons dans la dispersion des langues, dans le plaisir que fonde cette dispersion à partir de laquelle nous croyons être capables de restaurer un ordre civilisateur. La psychanalyse n'échappe pas à cette illusion culturelle lorsqu'elle croit interpréter un trauma fondateur, une première blessure, un rêve significatif qui semble amorcer de manière éloquente la trame d'une cure. Mais la psychanalyse, c'est l'apport majeur de Bion, situe le mythe de Babel comme une réponse douloureuse à l'incomplétude sexuelle du sujet.

${ }^{9}$ Paul Virilio, Esthétique de la disparition, Paris, Éditions Balland, 1980. 
Penser deviendrait alors l'objet d'une connaissance émotionnelle construite à partir du refoulement de la sexualité infantile. Penser permettrait de s'affranchir d'une mobilité pulsionnelle trop envahissante afin de privilégier le souhait d'une liberté aérienne; le sujet tenterait alors de justifier l'idéal d'une langue commune qui échapperait à la comuption, qui échapperait somme toute à la dispersion provoquée par un Surmoi divin.

Envisageant cette question, Bion demeure circonspect. II reconnaît certes que Babel est un mythe fondateur et que cette assise symbolique donne matière à penser. $\mathrm{Il}$ reconnaît au mythe une certaine universalité, comme Freud l'affirmait de son côté avec le mythe d'CEdipe. Mais Bion hésite entre le caractère fascinant du monde psychotique et l'intégrité scientifique du savant. En somme, Bion fait de la traduction l'élément paradoxal d'une pensée dont l'intégrité est revendiquée alors même que la dispersion est à l'ordre du jour.

Nous voilà au cceur même de la modernité que les mythes de Babel et d'Edipe introduisent de façon magistrale dans l'horizon culturel contemporain. Lorsque Bion fait valoir le caractère subversif de la curiosité - qui ne se confond pas chez ce dernier avec l'arrogance -, il affirme l'idée d'une connaissance revendiquée. Mais cette connaissance ne peut prendre forme que si l'action du refoulement, de l'interdit et de la transgression est à l'ordre du jour.

Il faut alors reconnaître dans la curiosité cette inscription du délit que connaît la communauté pour avoir enfreint la Loi de l'interdit. Edipe et la communauté de Babel partagent un même destin. Conséquence majeure de cette transgression :

L'exil et le refoulement, c'est-à-dire l'élément punitif dans les mythes, impliquent, en réalité, la création de limites, condition nécessaire sans laquelle il n'y a pas de connaissance possible. Paradoxalement, l'exil, la castration, le refoulement, l'aveuglement sont nécessaires car il est nécessaire, pour accéder à la connaissance, qu'il y ait une limite, une coupe assurant l'opportunité de se détacher de l'identique, de ce qui est 
pareil à soi-même, de l'indifférencié, de ce qui n'a pas de loi, une limite garantissant un ordre irréversiblement constitué. ${ }^{\text {It }}$

Nous pourrions ajouter que la dispersion des langues est intimement liée au monde psychanalytique. Se situer au cœur de Babel, c'est revendiquer face à un monde mythique révolu cette partition qui fait du sujet un être sexué, placé devant cette violente interrogation que lui offre le langage.

Université du Québec à Montréal

\section{Références}

AMATI MEHLER, J., ARGENTIERI, S., CANESTRI, J. (1994). La Babel de l'inconscient : langue maternelle, langues étrangères et psychanalyse. Paris, Presses Universitaires de France, coll. * Le fil rouge *.

ANZIEU, Didier (1992). Beckett et le psychanalyste. Paris, MenthaArchimbaud.

- (1989). * Beckett et Bion ". Revue française de psychanalyse : Bion. Tome LIII, 5 , septembre-octobre.

- (1987). Le penser : du Moi-peau au Moi-pensant. Paris, Dunod, Éditions Clancier-Guénaud.

- (1985). Le Moi-peau. Paris, Dunod, coll. « Psychismes n.

- (1983). " Un soi disjoint, une voix liante : l'écriture narrative de Samuel Beckett *. Nowvelle Revue de Psychanalyse.48, 1983.

- (1959). L'Auto-analyse de Freud. Paris, Presses Universitaires de France, coll. * Bibliothèque de psychanalyse $n$.

10 J. Amati Mehler, S. Argentieri, J. Canestri, La Babel de l'inconscient : langue maternelle, langues étrangères et psychanalyse, Paris, PUF, coll. * Le fil rouge ", 1994, p.19. 
BAIR, Deirdre (1979). Beckett, Paris, Fayard.

BECKETT, Samuel (1968). Watt. Paris, Éditions de Minuit.

- (1965). Murphy. Paris, Éditions de Minuit.

- (1951). Molloy, Paris, Éditions de Minuit.

BERMAN, Antoine (1984). L'épreuve de l'étranger : culture et traduction dans l'Allemagne romantique: Herder, Goethe, Schlegel, Novalis, Humboldt, Schleiermacher, Holderlin. Paris, Gallimard, coll. "Les Essais".

BlON, Wilfred Ruprecht $(1975,1977,1979)$. A Memoir of the Future. Book One : The Dream (1975); Book Two: The Past Presented (1977), Brazil, Imago Editoria; Book Three : The Dawn of Oblivion (1979). Pertshire, Clunie Press.

-(1970). Attention et interprétation. Paris, Payot, 1975.

- (1963). Elements of Psycho-Analysis. Londres, Heinemann, 1963.

-(1962), Learning from Experience. Londres, Heinemann, 1962.

- (1962). * A Theory of Thinking ". International Journal of PsychoAnalysis. 43, pp. 306-310.

- (1961). Recherche sur les petits groupes. Paris, Presses Universitaires de France, coll. “ Bibliothèque de psychanalyse », 1965.

- (1959). * Attacks on Linking *. Dans Second Thoughts. Londres, Heinemann, 167, pp. 93-109.

-(1950). " Le jumeau imaginaire *. Dans Réflexion faite. Paris, Presses Universitaires de France, coll. * Bibliothèque de psychanalyse „, 1983.

CASEMENT, P.J. (1982). * Samuel Beckett's Relationship to his Mother-Tongue ". International Review of Psychoanalysis. 9, 1982. 
CERTEAU, Michel de (1980). L'Invention du quotidien, t.l : Arts de Faire. Paris, 10/18, U.G.E.

CHIANTARETTO, Jean-François (1991). " Passages à l'écrit (à propos de l'écriture de la cure par l'analysant) ". Psychanalystes (Revue du Collège de psychanalystes). Paroles, écritures, 38.

- (1990). * Pour une approche psychanalytique de l'autobiographie ". Dans Psychanalyse à l'université. Paris, Presses Universitaires de France, 15,60 .

- (dir,) (1990). Le Coq-Héron. : « Autobiographie et psychanalyse. De la biographie à l'autobiographie $n, n^{\circ} 118$.

DAYAN, Maurice (1980). L'Arbre des styles, Paris, Aubier, coll. * La psychanalyse prise au mot ".

DOUBROVSKY, Serge (1988). Autobiographiques. De Corneille à Sartre. Paris, Presses Universitaires de France, coll. "Perspectives critiques ".

- (1980). Parcours critique. Paris, Galilée.

FUSCO, Marie-Claude (1977). "Faire part de son analyse ». Nowvelle Revue de Psychanalyse, « Écrire la psychanalyse ", n' 16, automne 1977.

GUILLAUMIN, Jean (1987). " Les enveloppes psychiques du psychanalyste w. Dans Les enveloppes psychiques (Didier Anzieu et al.). Paris, Dunod.

PACALY, Josette (1986). " De quelques récits de cure ». Cahiers de sémiotique textuelle, 8-9.

PEREC, Georges (1985). « Les lieux d'une ruse «. Dans Penser/Classer, Paris, Hachette.

RICGUR, Paul (1991). Temps et récit. 3 tomes, Paris, Seuil.

ROUSSEAU-DUJARDIN, Jacqueline (1995). L'imparfait du subjectif. 
Paris, L'Harmattan, coll. * La philosophie en commun n.

- (1993). Ce qui vient à l'esprit en situation psychanalytique. Paris, L'Harmattan.

- (1983). Couché par écrit. Paris, Galilée.

ROUSTANG, François (1980). ...Elle ne le lâche plus. Paris, Éditions de Minuit.

VIRILIO, Paul (1980). Esthétique de la disparition. Paris, Éditions Balland.

WINNICOTT, D.W. (1971). Playing and Reality. New York, Basic Books.

RÉSUMÉ : Écriture de soi et traduction dans les oeuvres * jumeiles " de Samuel Beckett et Wilfred R. Bion - Cet article envisage la problématique de la traduction à partir du champ psychanalytique. Wilfred R. Bion fut pour une brève période l'analyste de Samuel Beckett. L'auteur souhaite démontrer que les écrits de Samuel Beckett et Wilfred R. Bion peuvent être lus comme des cuvres jumelles qui tentent à leur manière de traduire l'infigurable source du « secret * de la cure. L'auteur aborde l'étude du concept d'après coup en psychanalyse, la mise en jeu du mythe de Babel dans l'ouvre théorique de Bion, la traduction de la langue-mère dans l'œuvre de Beckett. Les cuuvres de Bion et Beckett tenteraient ainsi l'impossible traduction du trauma dans la langue maternelle.

ABSTRACT : Writing the Self and Translation in the " Twin "Works of Samuel Beckett and Wilfred R. Bion - This article takes the psychoanalytic field as its point of departure in its discussion of the problematics of translation. For a brief period, Wilfred $R$. Bion was Samuel Beckett's analyst. The author endeavors to show that the writings of Beckett and Bion can be read as "twin" works that attempt, in their way, to translate the elusive source of the cure's "secret." The author examines the concept of après coup (deferred action) in psychoanalysis, the role of the Babel myth in Bion's theoretical writings, and the translation of the mother tongue in Beckett's writings. Both Bion and 
Beckett's writings would thus attempt the impossible translation, that of translating trauma into the mother tongue.

Simon Harel : Département d'études littéraires, Université du Québec à Montréal, C.P. 8888, Succ. A, Montréal (Québec) H3C 3P8. 